\title{
Systematic Approaches to Evaluation and Integration of Eastern and Western Medical Practices
}

\author{
Jan van der Greef, $\mathrm{PhD}{ }^{1,2}$ Herman van Wietmarschen, $\mathrm{PhD},{ }^{1,2}$ Yan Schroën, \\ Nathalie Babouraj, $\mathrm{MD}^{3}$ and Marion Trousselard, $\mathrm{MD}, \mathrm{PhD}^{4-6, *}$
}

\begin{abstract}
Background: The current health care system faces increasing costs and demands while the presence of chronic conditions in the general populations is rising. A shift from disease fighting toward health promotion is needed to prevent these conditions and empower people to work on their own health. In the Military setting, health promotion is equally important. Many Veterans are returning from missions with traumatic experiences. Therefore, in the field of mental health, it is also important to focus on building mental resilience before deployment, instead of starting treating after the damage is done. Throughout the ages, Eastern medicine systems have developed different views on health, disease, and resilience. These systems generally focus more on health promotion and strengthening the self-healing properties of the body, and are, therefore, complementary to Western medical developments. A better understanding and integration of those complementary views might reveal new avenues for treatments.

Objectives: In this chapter, Chinese Medicine, Ayurveda, and endobiogeny thinking are reviewed. Examples are then given of how systems biology can be applied to study Eastern medicine systems and reveal biologic information about certain diagnostic principles. These examples show how combined Western and Eastern diagnosis can be used to discover new patient subtypes. Those subtypes can then be used to study responses to medications and lifestyle interventions, leading toward personalized treatment in the future.

Results: Different studies open a field of exploration to combine systems biology into global health practice. This review shows that systems biology is successful in revealing biologic information about Eastern medicine practices. Conclusions: This new comprehensive approach, bridging the East and the West, creates novel opportunities for Military health care systems. More targeted and personalized treatment options can be developed for Soldiers, as well as novel health-promotion strategies based on foods, herbs, acupuncture, and other modalities.
\end{abstract}

Key Words: Systems Biology, Chinese Medicine, Ayurveda

\footnotetext{
${ }^{1}$ Principal Scientist and Scientist, respectively, TNO, The Netherlands Organization for Applied Scientific Research, Utrechtseweg, Zeist, The Netherlands.

${ }^{2}$ Chinese Medicine Practitioner and Scientist, Sino Dutch Centre for Preventive and Personalized Medicine, Utrechtseweg, Zeist, The Netherlands.

${ }^{3}$ Integrative Health Coach, Institut de Santé Intégrative, Montpellier, France.

${ }^{4}$ Manager, Neurophysiology of Stress Unit, Neuroscience and Operational Constraints, Département des Facteurs Humains, Institut de Recherche Biomédicale des Armées, Brétigny sur Orge, France.

${ }^{5}$ Physician and Neurosciences Researcher, Chaire Mindfulness, Bien-être au Travail et Paix Économique, Grenoble Ecole de Management, Grenoble, France.

${ }^{6}$ Professor in Cognitive Psychology, Ecole du Val de Grâce, Paris, France.

This article was prepared as part of the work product of NATO Science and Technology Organization, Task Force, Human Factors and Medicine (STO-TR-HFM) 195, Integrative Medicine Interventions for Military Personnel.

*The opinions and assertions contained herein are the private views of the author and are not to be construed as official or as reflecting the views of the French Armed Forces Biomedical Research Institute or the French Military Health Service at large. The author indicates that she does not have any conflicts of interests.
} 


\section{INTRODUCTION}

V ARIOUS CULTURES ACROSS THE GLOBE have given rise to a variety of sciences, resulting in different medical practices. Each of these medical systems makes use of particular concepts of health, wellness, resilience, and healing. In Western medicine, health is currently redefined as the ability to adapt and self-manage in the face of social, physical, and emotional challenges. ${ }^{1}$ In Chinese and Indian medicine, the concept of health is more related to being one with nature and the rhythms of life. An integration of medical systems is needed to take full advantage of the various viewpoints and enlarge our understanding of resilience, wellness, health, and the means of promoting health and resilience. ${ }^{2}$

Western and Asian medicine apply very different methods for assessing health. Western science is very good at measuring molecules and has generally followed a bottomup approach with regard to the pyramid of life described by Oltvai and Barabási. ${ }^{3}$ (Fig. 1). Elucidating higher levels of system organization is the concern of psychology. Overcoming the body-mind divide has proven to be very difficult. Knowledge of health and maintaining health is very limited, compared to the knowledge that has been gained about the molecular basis of diseases.

Asian medicine systems on the contrary have evolved as top-down approaches, phenomenological approaches, based on the organization and dynamics of symptom patterns. These dynamic symptom patterns consist of both physical as well as mental aspects, because the body-mind split has never occurred in Asian medicine. Therefore, Asian medicine provides an excellent opportunity to reconcile the body and mind in Western medicine. A half a decade ago, significant steps were taken to develop what is called a middle-out approach toward integration of measurements of these diverse levels of system organization. ${ }^{4}$

In this chapter, various cultures of medicine are described briefly, within a systems medicine context. Then, approaches to scientific evaluation and integration of various systems of medicine are discussed and illustrated with some examples from recent scientific literature.

\section{WESTERN AND EASTERN SCIENCES}

When we use the word science, there is no need for explanation. Every reader, anywhere in the world, will immediately assume that we are talking about the methodology used in the last 400 years and based on the ideas of Newton and Descartes, to try to understand our reality. Medicine is part of this scientific thinking. What science makes science are the rules that scientific philosophers such as Kühne and Popper have defined. However, what gave them the authority to devise these rules-that is, the embedding of their ideas in the Western cultural philosophy, which is rooted in our Western civilization?
If we were to conclude that there is only one science, the subsequent conclusion would also, necessarily, be that there is only one civilization in this world. If we were to assume that this was not the case, we could do the previous thinking exercise in reverse. Let us take a random civilization: the Chinese civilization. Do they have cultural philosophers? $\mathrm{Lao} \mathrm{Zi}$, Kong $\mathrm{Zi}$, Mo Zi.... So they have. Do they also have scientific philosophers? Yang Hui, Li Shizhen, Fung Yulan....Given that they do have scientific philosophers, who formulated ideas about how to approach and examine our reality? In addition, would it not be logical that these ideas were totally different from Western ones because of their different cultural bases? So, the Chinese approach to health and healing would also be different from our Western approach. To understand the methodology of Chinese medicine, we have to find a way to bridge the gap between the two paradigms ${ }^{4}$ (Fig. 2).

Chinese medicine is not object-oriented but is much more interested in the relationships between objects. In addition, Chinese medicine is much more focused on the quality then on the quantity of measurements. What Chinese medicine does is map patterns of relationships, relationships among symptoms, pulse characteristics, tongue features, and other factors. These are the exact characteristics of systems thinking - a new scientific way of thinking developed in the West after World War II, characterized by mapping relationships. Could this systems thinking be the bridge, the meta-language, between Western medicine and other systematic medical systems?

\section{AYURVEDA: A SYSTEMS SCIENCE}

Ayurveda, one of the oldest medical systems in the world, can bring new keys to understanding the complexity of health. Ayur means life in Sanskrit. Veda means science or knowledge. Ayurveda developed 3000 years BC and was organized into eight different branches: (1) general medicine; (2) pediatrics (including Cesarean sections for mothers); (3) psychiatric treatment; (4) ophthalmology (including cataract surgery); (5) surgery; (6) toxicology; (7) rejuvenation treatment (geriatrics); and (8) and sexology. Ayurveda is based on the body type of the individual that has to be taken into account, as well as the environment the person lives in, to determine a specific treatment for that individual patient.

According to Ayurvedic knowledge, the human being is the microcosmos, which is completely interconnected with his or her environment, the macrocosmos. Healthy relationships among every living system are primordial for global health, because everything is made of the five basic elements: Space; Air; Fire; Water; and Earth. In today's language, a rough translation would be: carbon; hydrogen; and oxygen atoms that are omnipresent in living and nonliving forms. 


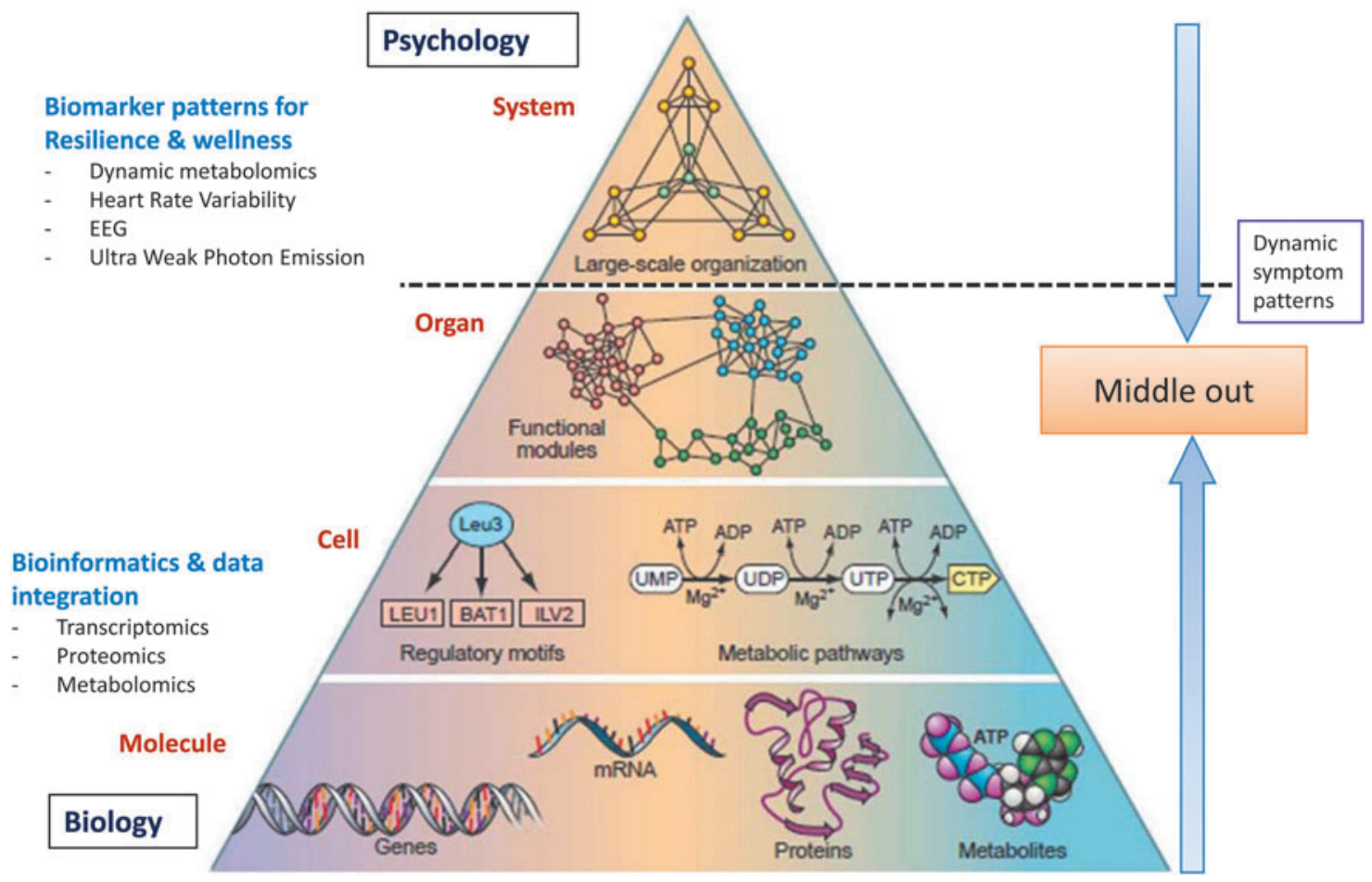

FIG. 1. A representation of levels of system organization, techniques for measuring these levels of organization, and the convergence of Chinese and Western science. EEG, electroencephalograph, LEU,; BAT,; ILV,; ATP, adenotriphosphate; ADP, adenosine diphosphate; UMP, uridine monophosphate; UDP, uridine diphosphate; UTP, uridine triphosphate; CTP, cytidine triphosphate. Adapted from Oltvai and Barabási, 2002, with permission from American Association for the Advancement of Science (AAAS).

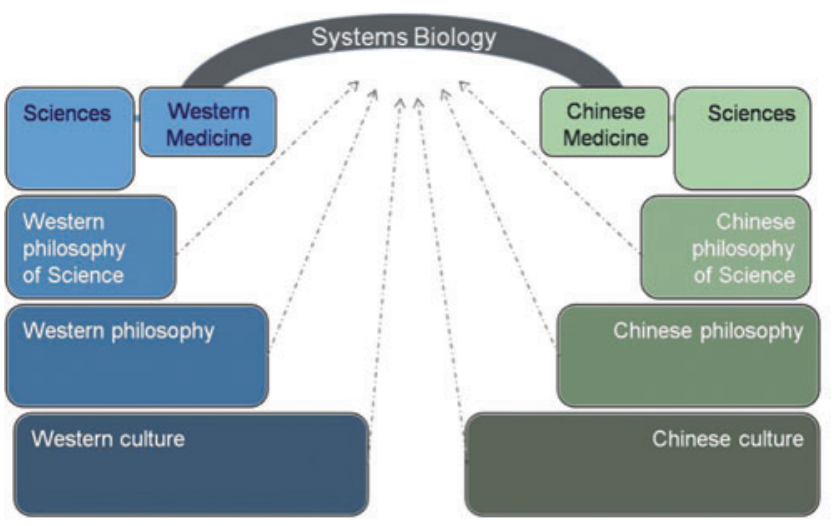

Origin of Man

FIG. 2. Systems biology as a bridge between Chinese and Western medicine. As migration moved populations apart in space and time, the Western and Chinese cultures evolved separately, giving rise to specific cultural philosophies. These philosophies gave rise to philosophies of science, which led to types of science. This evolution allowed different types of science to exist alongside one another. Figure reproduced from van der Greef, et al., 2010. Reprinted with permission from (C) Georg Thieme Verlag KG.
Ancient texts ${ }^{5}$ emphasize the importance of taking care of health before disease occurs, which would be proof of an imbalance of the body's original state. This is represented today by the term allostasis, which is explained in more detail in the following section. The aim of Ayurvedic treatment is to help a patient transition from a disease state to an allostatic state, or even to homeostasis, following the dynamic functional changes of the person's type.

It is worthwhile to focus a little bit more on the mechanisms of Ayurveda. An interesting view of these ancient texts is the concept that every human being is unique, and the physiology of each biologic system is ruled by forcesor doshas - that could be translated into functional phenotypes. In order to go a bit deeper into Ayurvedic diagnosis, it is helpful to focus on the doshas, or mind-body principle, that involve the five basic elements. Space and Earth give birth to the Vata dosha (Air); Fire and Water give birth to the Pitta dosha (Fire); and Water and Earth give rise to the Kapha dosha (Water). Everyone has a specific percentage of Fire-type (Pitta), a specific percentage of Water-type ( $\mathrm{Ka}$ pha) and a percentage of Air-type (Vata). It is during conception that human beings get their primary constitutions (Prakriti; Fig 3).

The force of Fire is responsible for all the digestive and metabolic processes of the body and the mind. The force of 


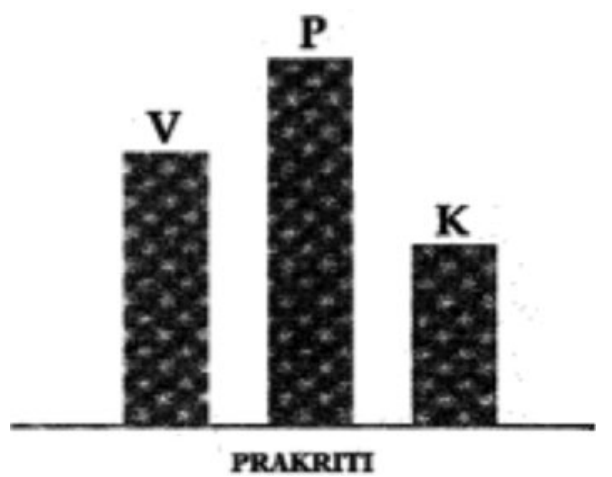

FIG. 3. The healthy situation is characterized by the right balance of the three forces: Wind $(\mathrm{V})$; Fire $(\mathrm{P})$; and Water $(\mathrm{K})$.

Water is the key to the structures of the body and the mind. Finally, the Air force is the guarantee for conduction and mobility in the body and the mind (digestion peristalsis, blood circulation, nerve impulses, breathing). When we translate this into a more physiologic process, every cell has these three forces it needs: structural components; metabolic capacity; and mobility capacity.

Other points are included in elaboration of the diagnosis, such as physical features (the quality of the tongue can also indicate dosha imbalances, as well as the study of the pulse, as in Chinese medicine). In addition to these physical semiologic features, an important part of the consultation is based on questioning regarding the patient's lifestyle and habits spanning his or her life.

With the years, and the effects of age and stressful situations, we divert from our Prakriti into a new state of imbalance known as Vikriti (Fig. 4). It is opportune for disease development. The initial percentages of the three functional forces are now disturbed - whether they are increased or decreased. The whole aim of the global treatment is to use herbal med-

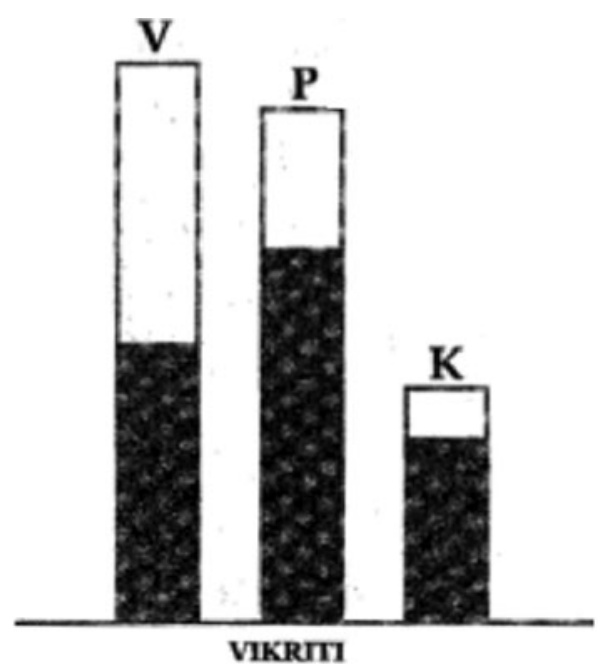

FIG. 4. The state in which the three forces-Wind (V); Fire (P); and Water $(\mathrm{K})$ - are out of balance. icines, massages, diet, yoga, and adaptation of the individual's environment to restore the Prakriti of the patient, in his or her five bodies, which are known as the five koshas ${ }^{6}$ (Fig. 5).

\section{THE SYSTEMS BIOLOGY APPROACH TO HEALTH IN EASTERN MEDICINE}

In systems biology, an organism can be considered to be a dynamic, self-organizing system. ${ }^{7,8}$ Not only the physiology and anatomy of the human body can be approached in this fashion - pathology also appears to be dynamically organized.

Symptoms arise in groups and create a dynamic balance. In Chinese medicine, it is said that symptoms group around virtual centers, for which poetic names, such as Spleen $Q i$ Deficiency or Liver Yang Rising, are used in Chinese diagnosis. Ayurvedic, Tibetan, and other Asian medicine systems have their own sets of names for such virtual centers. However, these labels should not distract us, although these exotic names are far from our Western scientific reality. Statically, we can see that the grouping of the symptoms is very consistent. In addition, if a symptom appears that does not primarily belong to a stable group of symptoms, the system tends to behave chaotically. Such a symptom is called a "bridge symptom." It brings the dynamically balanced system of a diseased body into a new dynamic steady state in which the patient can feel better or worse.

This could indicate that health is a dynamic state in which an organism's system responds between a maximum and minimum: the homeostatic state. Actually, we could say that a person is at maximum health when that person's dynamic
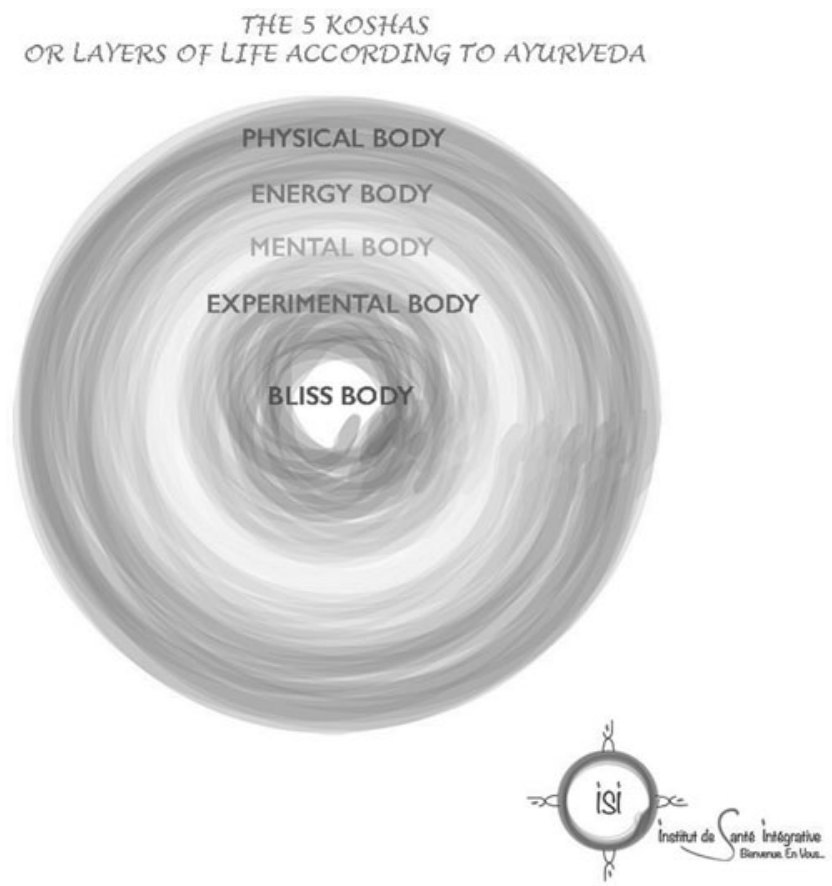

FIG. 5. The five bodies or five koshas according to Ayurveda. 
self-organized system is as open as possible and exchanges the largest possible amount of information with its environment, without losing its identity.

When the system is challenged, it can go into an allostatic state in which the dynamic system is stretched to a maximum but can recuperate by itself. When the system is frequently challenged to the allostatic state, the system can adapt to that state and become part of the homeostatic state. When the system is challenged to a level that it cannot recuperate by itself, the system goes into a disease level. The system organizes itself into a new dynamic balance, which is not perceived as pleasant or healthy by the patient. When the system is not helped to make the move back into the allostatic or homeostatic state, the system will move into a next disease level (Fig. 6).

\section{SYMPTOMS AS INTERMEDIARIES BETWEEN WESTERN AND ASIAN DIAGNOSES}

A Chinese syndrome is characterized by a specific pattern of symptoms, just like a Western disease is characterized by a specific group of symptoms. However, Chinese syndromes and Western disease patterns are determined by different sets of diagnostic tools. Chinese diagnosis makes use of questioning a patient, observing the tongue, and taking the pulse, among other ways of obtaining information about the patient. Western medicine developed many measurement techniques to identify changes in, for instance, blood parameters. A Western disease can consist of many different Chinese syndromes. Conversely, a Chinese syndrome can belong to many different Western diseases. In this way, symptoms can bridge the Western and Chinese diagnoses and help us subtype Western diseases and find new relationships among the different Western diseases we have not been able to observe yet. Discovering relevant subtypes of diseases, such as rheumatoid arthritis (RA) and type 2 diabetes mellitus is very important for optimizing treatment strategies and moving toward personalized medicine approaches.

Figure 7 shows an example of this approach in which RA symptoms were compared to symptoms of Chinese syndromes. In addition, the symptoms of some other rheumatic diseases were also compared to the same Chinese syndromes. This resulted in a network of relationships as shown in the figure, showing that RA is indeed related to many Chinese syndromes. After checking these observations with Chinese medicine experts, a combination of two general TCM subtypes with RA was chosen for further study, Cold RA and Heat RA. Another argument for this choice was that those two subtypes of patients with RA are treated very differently in Chinese medicine. Therefore, great differences in disease mechanisms can be expected as well as differences in responses to Western medications.

\section{METABOLOMICS TECHNOLOGY FOR VALIDATING AND UNDERSTANDING SYMPTOM PATTERNS}

An approach was needed to build a bridge between symptom patterns observed and used in Asian medicine and the molecular biologic approach on which Western medicine is founded. The Netherlands Organization for Applied Scientific Research (TNO), Utrechtseweg, Zeist, The Netherlands,

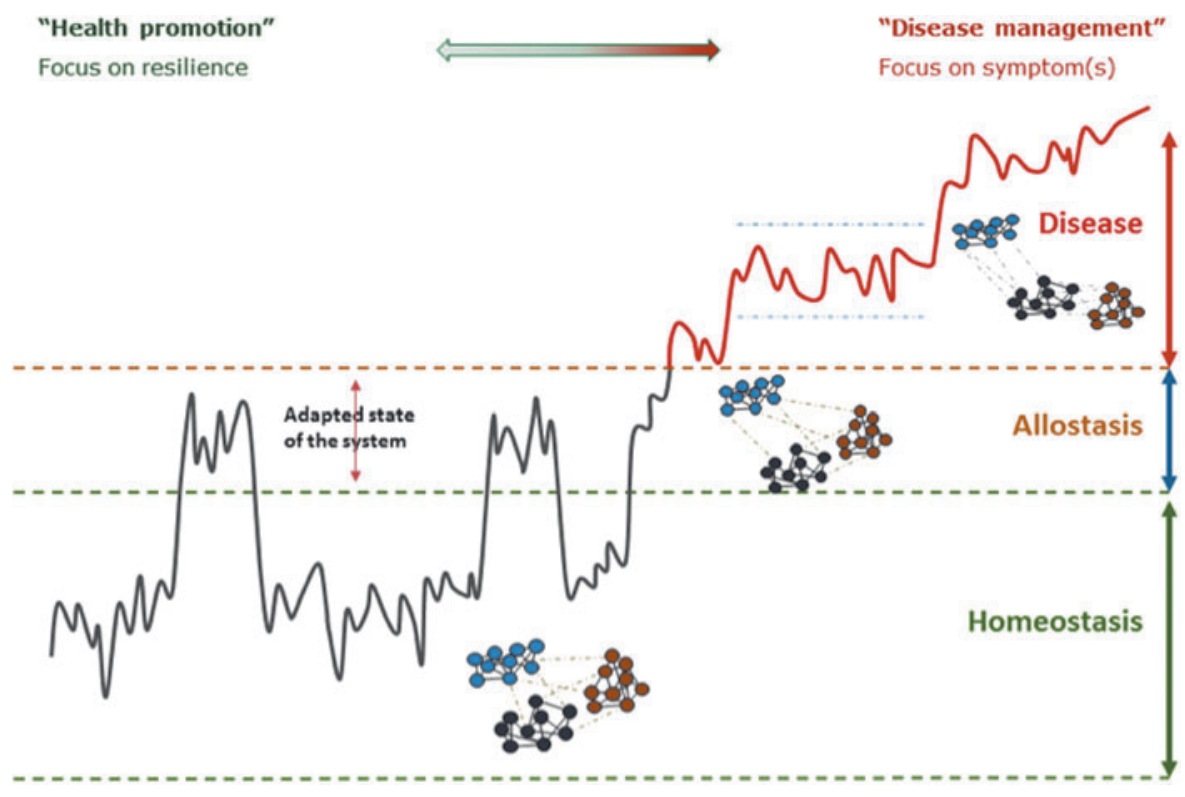

FIG. 6. Conceptual positioning of the relationships among health, resilience, and allostasis. Health is challenged by acute stressors and by chronic stressors that could build up allostatic load over time. The response to sudden challenges is often conceptualized as resilience. 


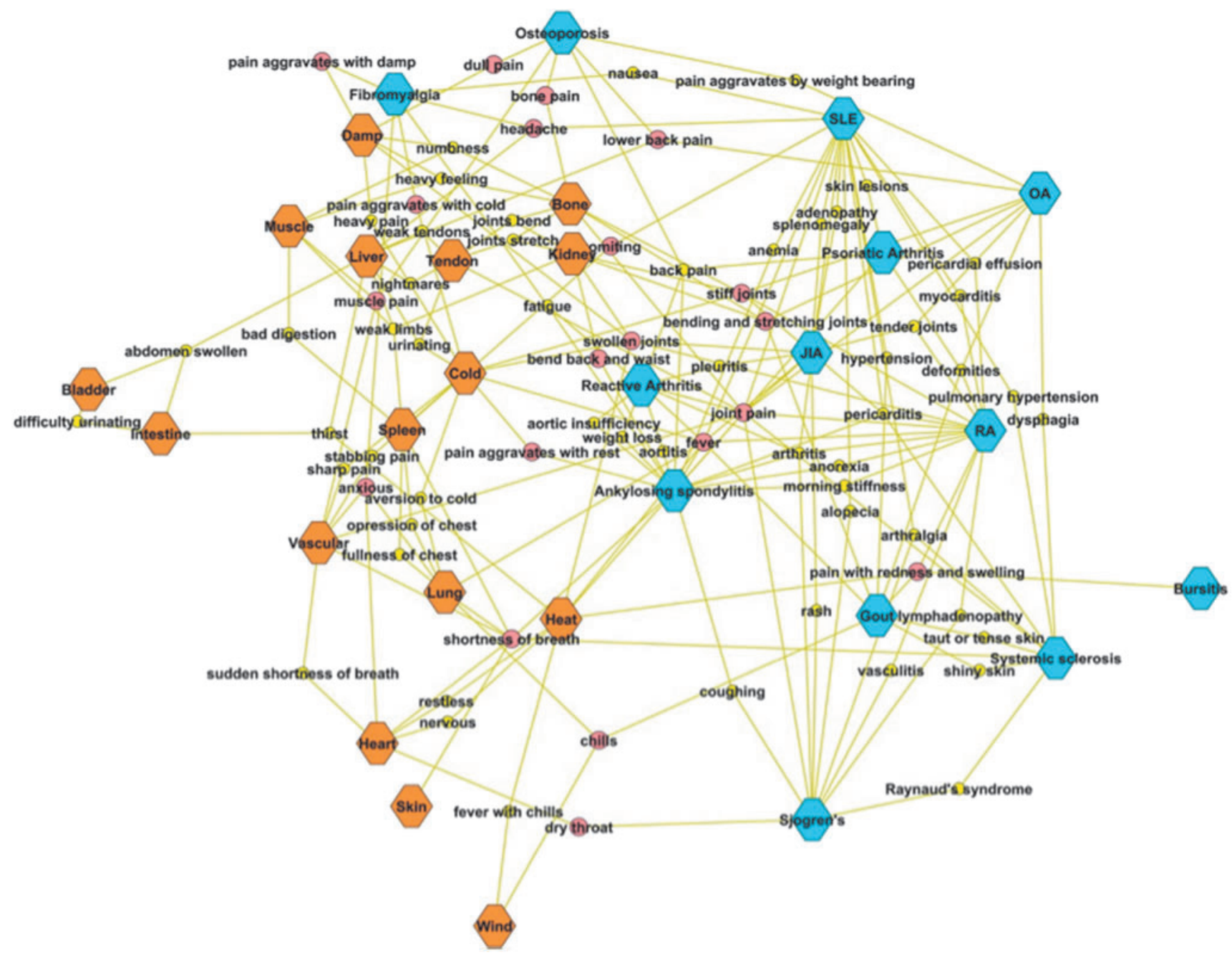

FIG. 7. Overlapping symptom patterns between Western rheumatic diseases and Chinese arthritis related syndromes. The blue hexagons represent the Western disease categories while the orange hexagons represent the Chinese syndromes. The small circles represent symptoms. Only those symptoms are shown that are representative of at least two syndromes, diseases or disease/syndrome combinations (bridge symptoms). SLE, systemic lupus erythematosus; OA, osteoarthritis; JIA, juvenile idiopathic arthritis; RA, rheumatoid arthritis.

together with the Sino Dutch Centre for Preventive and Personalized Medicine, Utrechtseweg, Zeist, The Netherlands, pioneered two research lines in which metabolomics was used to characterize patient subtypes. ${ }^{9}$ Western patient classes, RA, and type 2 diabetes, were subtyped using Chinese diagnosis. Then blood and urine samples were collected from these patients for metabolomics analysis with the aim of finding biologic molecular mechanisms behind the Chinese subtypes.

Figure 8 summarizes the systems biology approach in which metabolomics is one of the technologies that can be complemented by others, such as proteomics and transciptomics. Metabolomics focuses on measuring metabolites, which represent the state of the body at a certain moment and can give indications of many processes, such as inflammation, energy balance, oxidative stress, metabolism, hormone organization, etc.
Analysis of the data is a critical step. Various multivariate data analysis techniques especially suited to working with metabolomics data have been developed over the years. More than a decade ago, nonlinear data analyses had begun to evolve in order to supplement the linear ones. ${ }^{10}$

Two subtypes of RA, RA Cold and RA Heat, could be classified based on symptom and clinical chemistry profiles with a classification error of $15 \%$. The same patients could also be classified based on urine metabolite profiles with a classification error of $14 \% .^{11}$ Figure 9 shows how the results of a principal component analysis are presented. In the left panel, differences between patients with RA Cold and RA Heat are shown. Both groups are almost separated, but there is some overlap. In the right panel, the symptoms and clinical chemistry measurements that are responsible for most of the variation in the patient groups are 


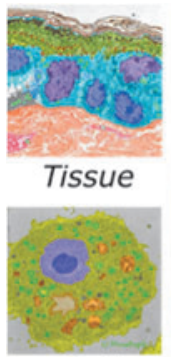

Cell

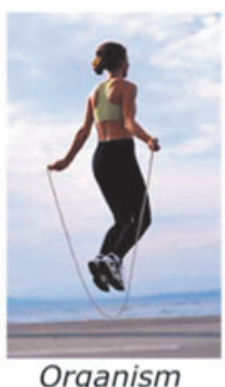

Organism

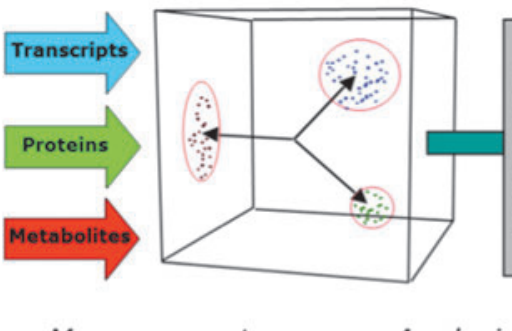

Measurement

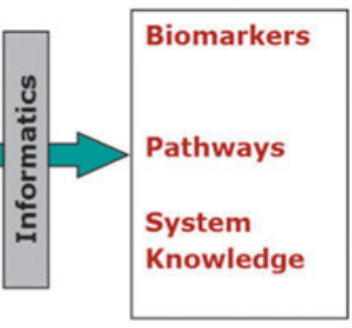

Interpretation

FIG. 8. Systems biology approach involves measuring large numbers of metabolites from various tissues and cells. Next, the approach involves using multivariate data analysis techniques to determine the most relevant variables, which are subsequently used for interpretation.

shown. The ones pointing toward the left are most related to RA Cold, and those pointing toward the right are most related to RA Heat.

A similar study was performed with patients with prediabetes who were diagnosed by three TCM practitioners in three patient groups: (1) Qi and Yin Deficiency; (2) Qi and Yin Deficiency with Dampness; and (3) Qi and Yin Deficiency with Stagnation. Metabolomics measurements of urine samples revealed differences in carbohydrate metabolism and renal function between the Stagnation group and the other two groups ${ }^{12}$ (Fig. 10).

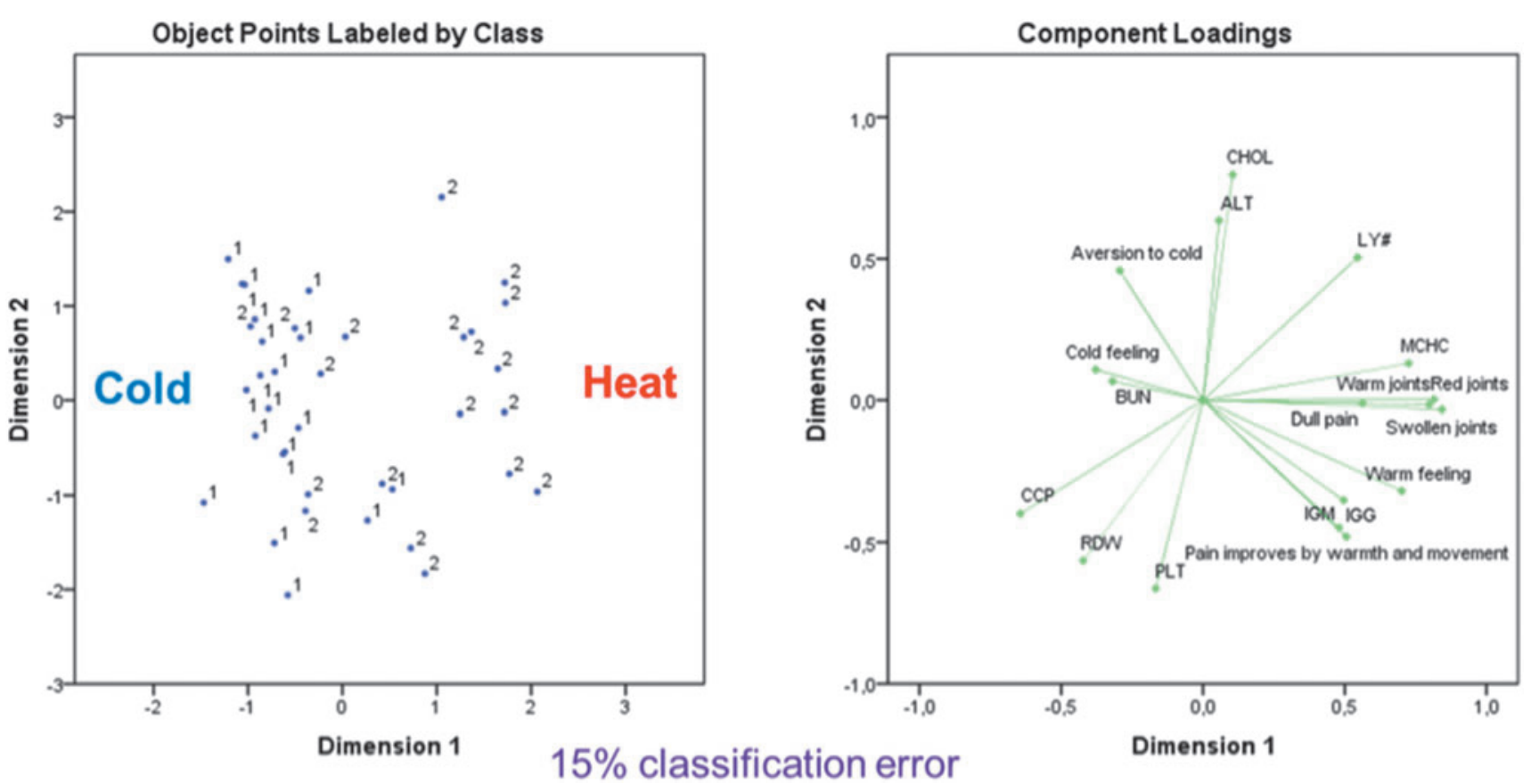

FIG. 9. The left panel shows the scores of the patients with Cold and Heat rheumatoid arthritis (RA) obtained by the optimal principal component analysis principal component analysis model. The right panel shows the loadings of the variables used in the PCA model. The variables pointing toward the right are mostly related to the Heat RA group while the variables pointing toward the left are related to the Cold RA group. CHOL, cholesterol; ALT, alanine aminotransferase; LY\#, lymphocyte number; MCHC, mean corpuscular hemoglobin concentration; BUN, blood-urea-nitrogen; CCP, citrullinated protein antibodies; RDW, red blood cell distribution width; PLT, platelet count; IGM (IgM), immunoglobulin M; IGG (IgG), immunoglobulin G).

Similar to the TCM/systems biology studies on RA Heat and RA Cold, it is possible to define subtypes based on Ayurvedic symptoms, involving Ayurvedic symptom patterns matching with Western rheumatoid arthritis. ${ }^{13}$

According to presence or absence of Dampness, the patient will be Kapha- aggravated (excess of Water) or Kapha-lacking. The presence of symptoms of Pitta (inflammation) will make the patient Pitta-positive or Pitta-negative, and dryness will be a sign of Vata aggravation. If subgroups of patients could be recruited, their specific metabolomic profiles could be measured, conventional treatment plus the specific Ayurvedic 


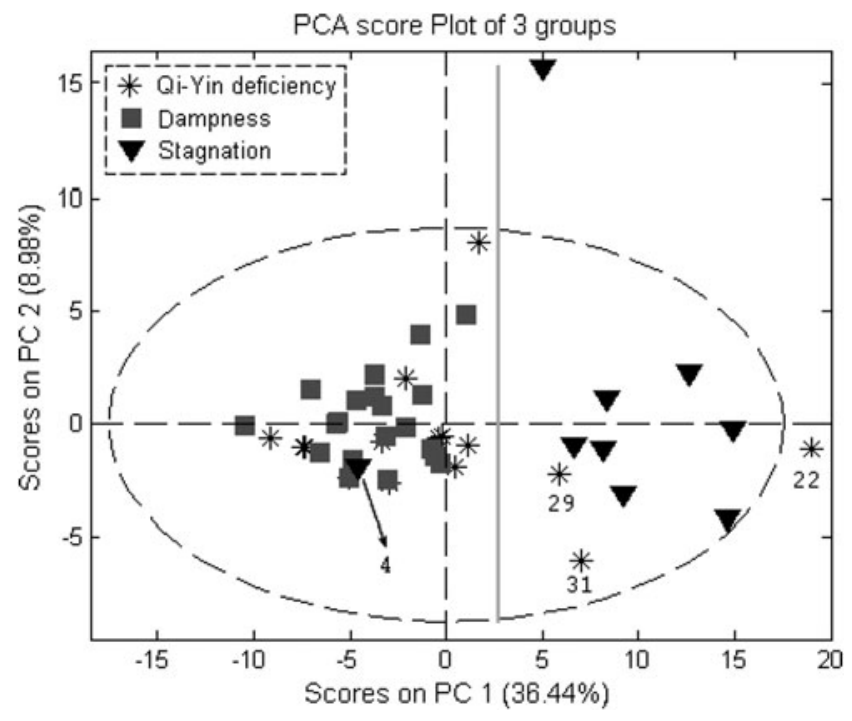

FIG. 10. Principal component analysis score plot for three groups of patients with prediabetes. The Stagnation group is separated from the Dampness group, indicating that different concentrations of urine metabolites have been measured for those subgroups. PC1, principal component 1 .

corrections could be given, and the evolution of the patients' symptoms could be followed. Many research teams focusing on this field of Ayurgenomics are finding interesting patterns for use in preventive medicine. ${ }^{14}$ An example of subtyping chronic back pain after a Military mission is shown in Figure 11.

\section{ENDOBIOGENY: A SYSTEMS ENDOCRINOLOGY APPROACH}

Another interesting systems biology approach is endobiogeny. It was developed by French medical physicians, Jean-Claude Lapraz, MD and Christian Duraffourd, MD in the early 1970s. ${ }^{15}$ Endobiogeny's focus is on the relationships among the different endocrine regulation loops and predicting the stage of an individual (homeostasis, allostasis or disease) according to various indexes reflecting the endocrine functions (Fig. 12).

Each function is quantified by an index, specified by a level of activity, and qualified by a score. The index expresses the resulting effectiveness of the function's activity, both in itself and in relation to the metabolic needs of the organism. The whole set of indexes gives a very precise longitudinal assessment of an individual body's functionality, system by system, organ by organ.

These indexes are calculated mostly from commonly used blood analysis data using formulae that reflect the modalities of functionality. Reliability and reproducibility are provided by a computer-based model that simultaneously determines the whole set of indexes.

This biology of functions allows one to determine the pathogenic tendencies of an organism, its stage of development, and the degree of severity of a potential pathology. Biology of functions can also be used as a tool to track the natural development of the pathology and its development

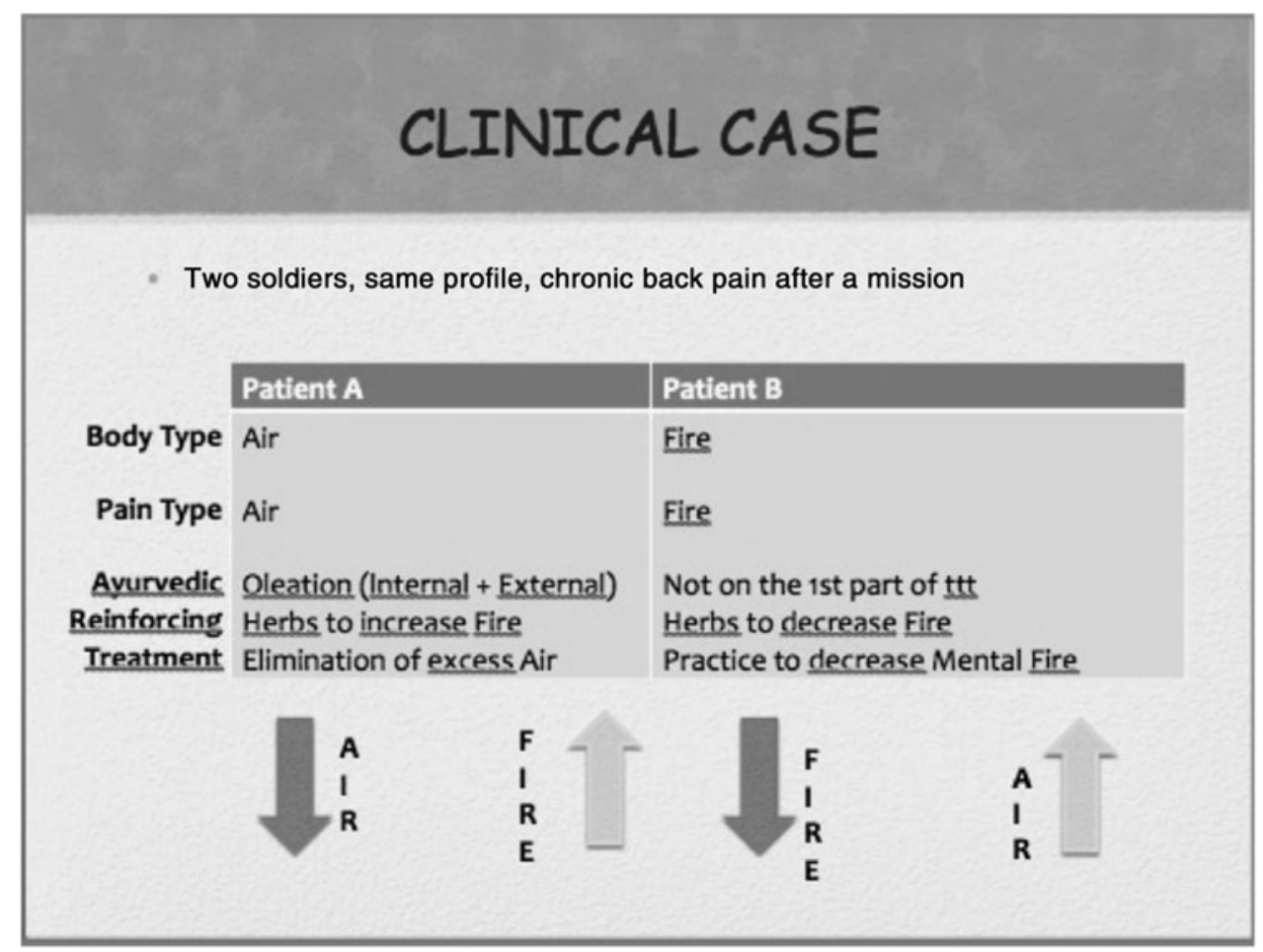

FIG. 11. A simplified clinical case including conventional Western diagnosis and Ayurvedic specificities for chronic low-back pain. $\mathrm{ttt}$, treatment. 


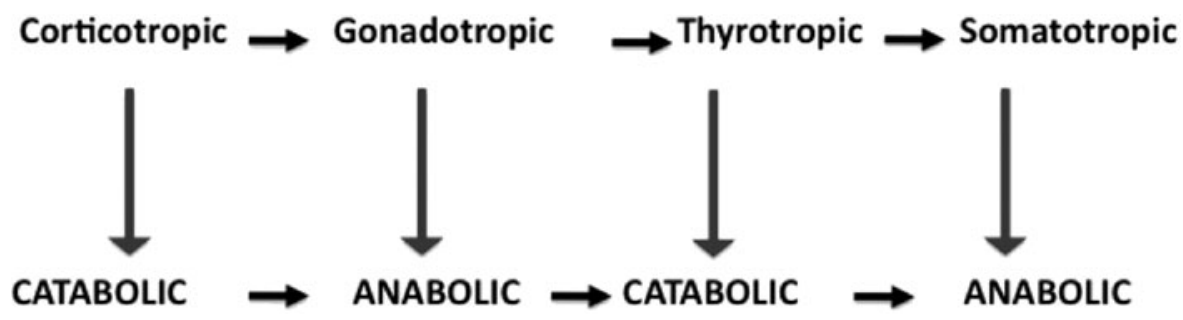

FIG. 12. Relationships among the endocrine functions. Metabolism begins with catabolism and ends with anabolism. The various axes alternate in their metabolic effects and complement each other by starting or ending the activity of the axis that precedes an axis in a continuous loop of metabolic activity.

under treatment, in order to adjust the latter better. A flow chart of a study conducted within the French military is presented in Figure 13.

\section{MODELING INTEGRATIVE MEDICINE PERSPECTIVES}

An essential element for integration of the various types of information obtained from Western scientific approaches and Asian scientific approaches is a method for modeling relationships and dynamics. ${ }^{16}$ Mechanistic modeling can be useful in this integration, because mechanistic modeling provides the means to connect both approaches. Suppose we have a model that describes some physiologic variables that are also used for diagnosis in Chinese medicine. Such a model can be extended with a set of variables that describe certain aspects of health from a Chinese perspective, based on a set of equations or rules. Simulations for a number of well-chosen conditions can then be used to display, side by side, the effects of these conditions on Western and Chinese indicators of health. This enables mutual understanding and a common ground for discussions about, and exchanges of, knowledge between Western and Chinese experts.

Figure 10 shows an example of how the above can work in practice. TNO developed a first prototype model of a systems health model that describes various aspects of health, including glucose metabolism, mental stress, and inflammation. The inputs of the model are factors related to lifestyle, such as food intake, exercise, and sleep. This model was built in MARVEL, a modeling tool that combines a relatively simple representation of the system of interest $^{17}$ with an easy-to-use interface. The interface allows the use of a touch table in interactive discussion sessions with domain experts and stakeholders.

Although a simple representation has some drawbacks, it also has a number of clear advantages: it can intuitively be understood by people who are not familiar with modeling

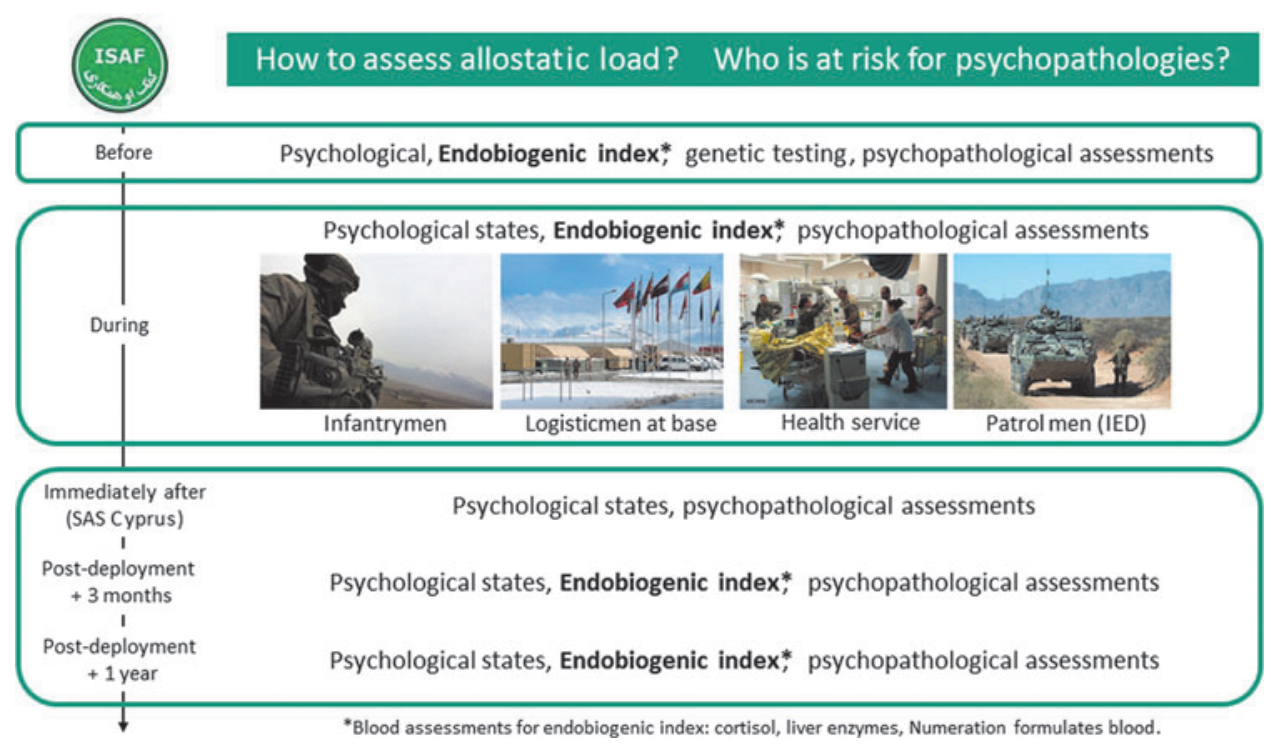

FIG. 13. Flowchart of an endobiogeny study conducted in the French military. ISAF, International Security Assistance Force; IED, improvised explosive device; SAS, Special Air Service. 
and it allows fast simulation of the effects of changes in input variables, allowing for vivid interaction with experts. Panel A of Figure 14 shows the variables and their interactions (the shapes of the arrows in the figure indicate strengths and speeds or interactions). The simulation view in Panel B shows the dynamics of a simulation in which cumulative overfeeding leads to the onset of type 2 diabetes. The radar chart in Panel $\mathrm{C}$ provides an alternative visualization of cause-effect relationships derived from simulation results.

\section{A}
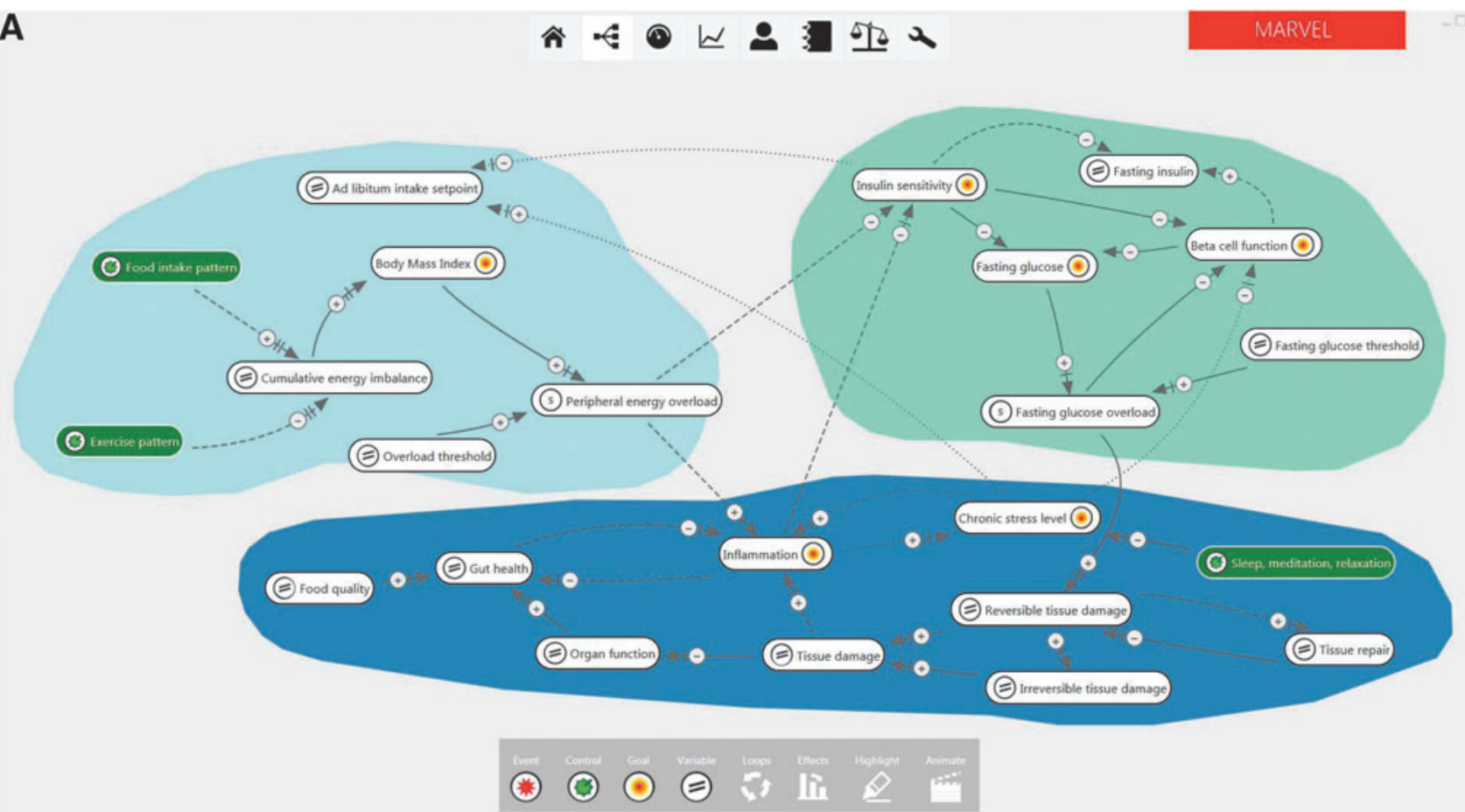

B

B

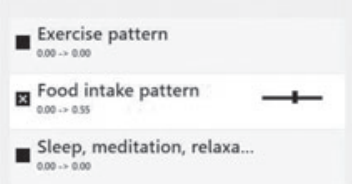

- Show neutral variables

- Show controls

\Show goals

Whow radar chart

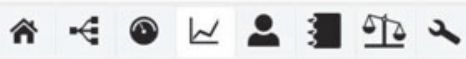

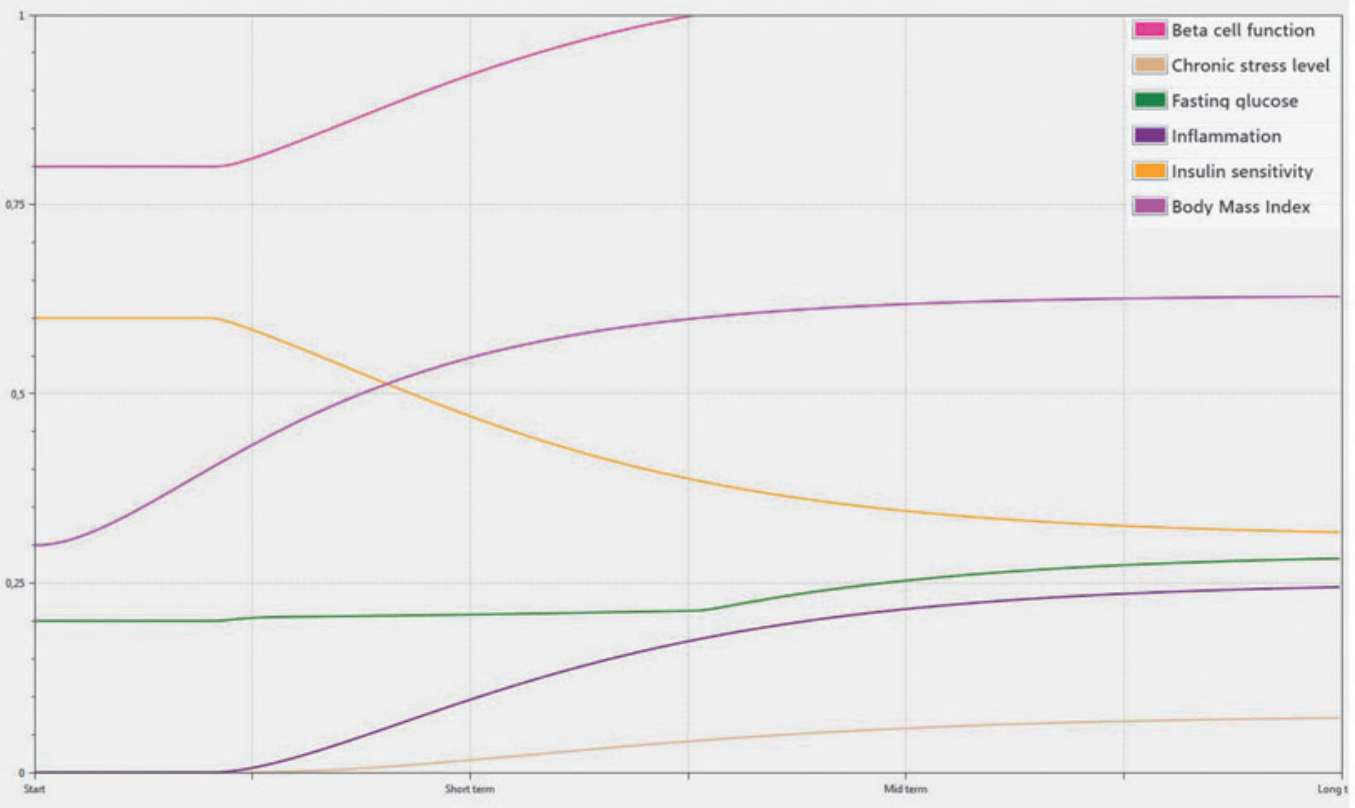

FIG. 14. Prototype systems health model built in MARVEL. Panel A shows the variables and their interactions (shapes of arrows indicate strengths and speeds or interactions). Panel B shows a simulation of the dynamics in which cumulative overfeeding leads to the onset of type 2 diabetes. The radar chart in Panel $\mathbf{C}$ provides an alternative visualization of cause-effect relationships derived from simulation results. 


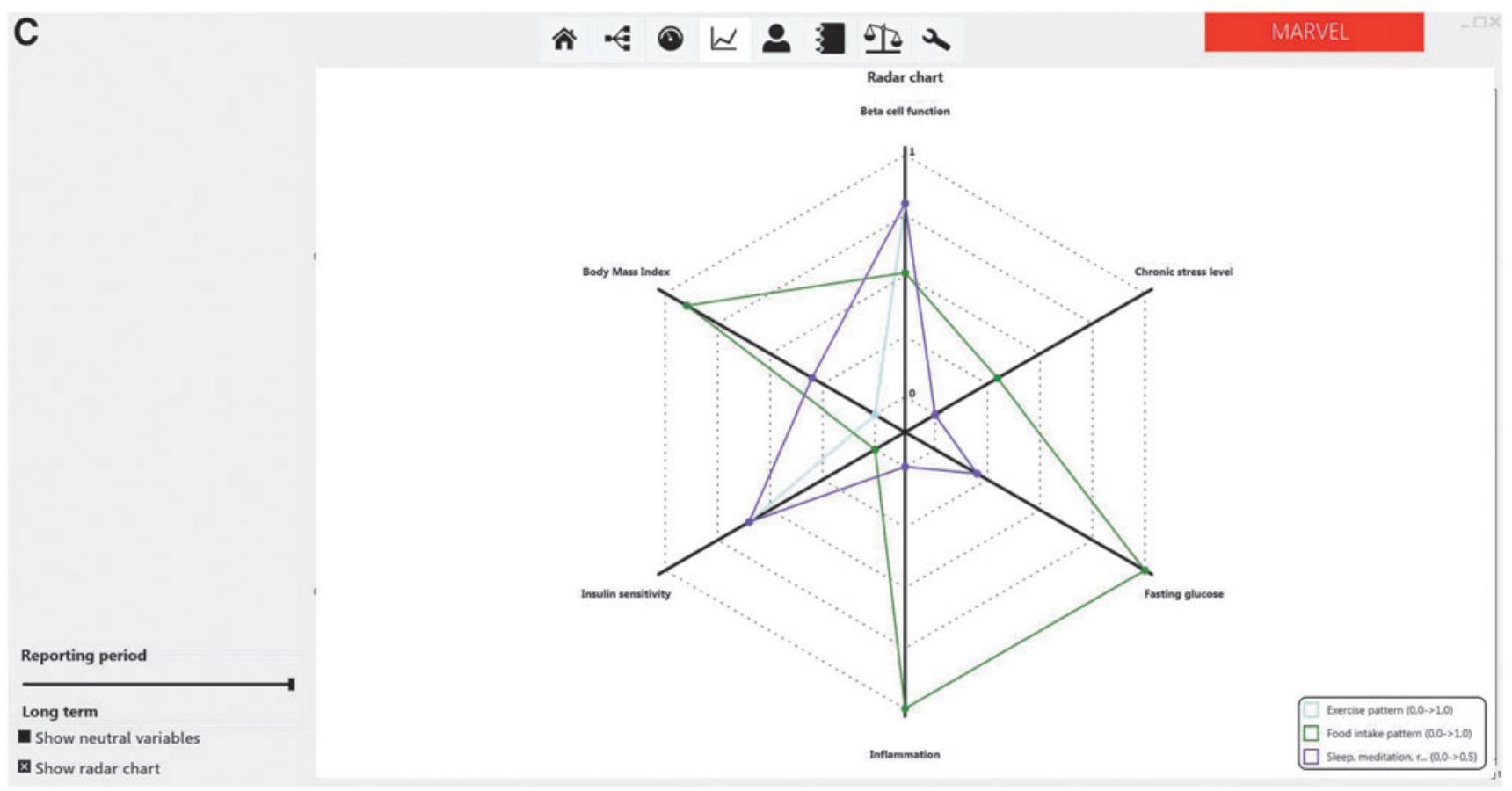

FIG. 14. (Continued).

\section{CONCLUSIONS}

This new comprehensive approach, bridging the East and the West, creates novel opportunities for Military health care systems. More targeted and personalized treatment options can be developed for Soldiers, as well as novel health-promotion strategies based on foods, herbs, acupuncture, and other modalities.

\section{RECOMMENDATIONS FOR FUTURE RESEARCH}

Asian science and medicine is essential for the future of health care. There is a clear need to bridge the chasm between psychology and biology, which appears to be marginally successful in current scientific approaches. Asian medicine can provide an integrative perspective on many diseases, allowing opportunities for better treatment and more personalized treatment.

Two essential focus points can be identified to move the field forward from a scientific point of view. First, techniques should be developed further to obtain Asian symptom patterns from patients in an objective manner. This involves a shift from developing questionnaires, which are linear in nature, toward developing expert systems. Expert systems are ideally suited to address the nonlinear nature of gathering patient information and discovering underlying disease patterns. Such expert systems can be installed easily on mobile devices for use in the field.
Second, approaches should be developed to integrate the variety of types of data using state-of-the-art dynamic mechanistic modeling techniques. Effort needs to be made to develop such models with currently available knowledge in the literature and from experts.

Both of the above elements are relevant in a Military setting. The expert systems based on Asian dynamic symptom patterns provide a unique diagnostic system for tracking aspects of resilience, fitness and health. The dynamic models can also be installed on mobile devices, enabling tracking of many sources of data, such as food intake, activity patterns, physiologic measures, mood, etc. The model can then be used to simulate the effects of possible interventions on the performance of an individual. Such models can therefore be used by an individual but also by commanders for monitoring unit resilience. At many levels of a Military organization, these tools can provide hypotheses for interventions, directions for spending research money, tracking resilience and health, and empowerment for individuals.

\section{AUTHOR DISCLOSURE STATEMENT}

Jan van der Greef, $\mathbf{P h D}$, is a principal scientist working in the field of systems biology at TNO, for the Sino-Dutch centre for Preventive and Personalized Medicine and as a professor at the Analytical Chemistry department at Leiden University. He was appointed as a member of the NATO panel HFM-195 ("Integrative Medicine Interventions for Military Personnel"). TNO resources were used to support 
travel expenses to the team meetings. The author did not receive any funding by governmental or non-governmental organizations to write this report. No competing financial conflicts exist. Herman van Wietmarschen, PhD, is a scientist working in the field of systems biology at TNO and for the Sino-Dutch centre for Preventive and Personalized Medicine, and was a guest invited to present at two NATO panel HFM-195 ("Integrative Medicine Interventions for Military Personnel") meetings. TNO resources were used to support travel expenses to the team meetings. The author did not receive any funding by governmental or nongovernmental organizations to write this report. No competing financial conflicts exist. Yan Schroën is a Chinese medicine practitioner with a clinical practice and working as a scientist for the Sino-Dutch centre for Preventive and Personalized Medicine (SDPPM). He as invited as a guest speaker to present at two NATO panel HFM-195 ("Integrative Medicine Interventions for Military Personnel") meetings. SDPPM resources were used to support travel expenses to the team meetings. The author did not receive any funding by governmental or non-governmental organizations to write this report. No competing financial conflicts exist. Nathalie Babouraj, MD, was at the time of the group meetings an Active Duty Service Member in the French Army, and was appointed to the NATO panel HFM195 ("Integrative Medicine Interventions for Military Personnel") as a technical team member for the duration of the Team. Resources from the French Ministry of Defense supported the travel expenses to the team meetings. The author did not receive any funding by governmental or nongovernmental organizations to write this report. The author was never employed or paid by any military or religious organization. She is now a civilian. No competing financial conflicts exist. Marion Trousselard, MD, PhD, is an Active Duty Service Member in the French Army, and was appointed to the NATO panel HFM-195 ("Integrative Medicine Interventions for Military Personnel") as a technical team member for the duration of the Team. Resources from the French Ministry of Defense supported the travel expenses to the team meetings. The author did not receive any funding by governmental or non-governmental organizations to write this report. The author was never employed or paid by any military or religious organization. No competing financial conflicts exist.

\section{REFERENCES}

1. Huber M, Knottnerus JA, Green L, et al. How should we define health? BMJ. 2011;343:d4163-d4163.

2. van der Greef, J. Perspective: All systems go. Nature 2011; 480(7378):S87-S87.

3. Oltvai ZN, Barabási AL. Systems biology. Life's Complexity pyramid. Science. 2002;298(5594):763-764.

4. van der Greef J, van Wietmarschen H, Schroën J, Wang M, Hankemeier T, Xu G. Systems biology-based diagnostic principles as pillars of the bridge between Chinese and Western medicine. Planta Med. 2010;76(17):2036-2047.

5. Valiathan MS. The Legacy of Caraka. Himayatnagar, Hyderabad, India: Orient Blackswan Private; 2003.

6. Garodia P, Ichikawa H, Malani N, Sethi G, Aggarwal BB. From ancient medicine to modern medicine: Ayurvedic concepts of health and their role in inflammation and cancer. J Soc Integr Oncol. 2007;5(1):25-37.

7. Dallmann R, Viola AU, Tarokh L, Cajochen C, Brown SA. The human circadian metabolome. Proc Natl Acad Sci U S A. 2012;109(7):2625-2629.

8. Glass L. Sychronization and rhythmic processes in physiology. Nature. 2001;410(6825):277-284.

9. van der Greef J, van Wietmarschen H, van Ommen B, Verheij E. Looking back into the future: 30 years of metabolomics at TNO. Mass Spectrom Rev. 2013;32(5):399-415.

10. Meulman JJ, van der Kooij AJ, Heiser WJ. Principal components analysis with nonlinear optimal scaling transformations for ordinal and nominal data. In: Kaplan DW, ed. The SAGE Handbook of Quantitative Methodology for the Social Sciences. Thousand Oaks, CA. Sage Publications; 2004:49-70.

11. van Wietmarschen H, Yuan K, Lu C, et al. Systems biology guided by Chinese medicine reveals new markers for subtyping rheumatoid arthritis patients. J Clin Rheumatol. 2009; 15(7):330-337.

12. Wei H, Pasman W, Rubingh $\mathrm{C}$, et al. Urine metabolomics combined with the personalized diagnosis guided by Chinese medicine reveals subtypes of pre-diabetes. Mol Biosyst. 2012; 8(5):1482-1491.

13. Chopra A, Lavin P, Patwardhan B, Chitre D. A 32-week randomized placebo-controlled clinical evaluation of RA-11, an Ayurvedic drug, on osteoarthritis of the knees. J Clin Rheumatol. 2004;10(5):236-245.

14. Dey S, Phawa P. Prakriti and its associations with metabolism, chronic diseases, and genotypes: Possibilities of new born screening and a lifetime of personalized prevention. J Ayurveda Integr Med. 2014;5(1):15-24.

15. Lapraz JC, Hedayat KM, Endobiogeny: A global approach to systems biology (part 1 of 2). Glob Adv Health Med. 2013; 2(1):64-78.

16. De Graaf AA, Freidig AP, De Roos B, et al. Nutritional systems biology modeling: From molecular mechanisms to physiology. PLoS Comput Biol. 2009;5(11):e1000554.

17. Zijderveld E. MARVEL_Principles of a Method for SemiQualitative System Behaviour and Policy Analysis [presentation]. System Dynamics Society Conference. Boston; (2007:1-21). Online document at: http://systemdynamics.org/ conferences/2007/proceed/papers/VAN\%20Z213.pdf Accessed September 14, 2015.

Address correspondence to: Herman van Wietmarschen, $P h D$ Sino-Dutch Centre for Preventive and Personalized Medicine P.O. Box 360 3700 AJ, Zeist The Netherlands

E-mail: herman.vanwietmarschen@tno.nl 